\title{
ESTADO EMOCIONAL DEL INTERNO ANTE LA PRIVACIÓN DE LIBERTAD
}

\author{
Marta Megía Ciudad \\ Educadora Social \\ martamegiaciudad@gmail.com \\ Ana Isabel Isidro de Pedro \\ Universidad de Salamanca \\ anyis@usal.es
}

Recepción Artículo: 27 octubre 2021

Admisión Evaluación: 27 octubre 2021

Informe Evaluador 1: 28 octubre 2021

Informe Evaluador 2: 29 octubre 2021

Aprobación Publicación: 30 octubre 2021

\section{RESUMEN}

La prisionalización requiere una adaptación, tanto física como psicológica, que el interno en una institución penitenciaria debe realizar para su adecuación al contexto carcelario puesto que, al verse privado de libertad, se produce un cambio sumamente brusco en su vida. Así, muchos y variados son los efectos psicológicos que padecen los sujetos tras un periodo de tiempo residiendo en una institución penitenciaria. En tal sentido, este estudio pretende analizar los efectos de la prisionalización, fundamentalmente en cuanto a los estados emocionales que suscita en el individuo y el impacto que genera esta experiencia en su desarrollo. Para ello, se ha utilizado un cuestionario ad hoc de elaboración propia, conformado por 46 ítems (de opción múltiple, tipo Likert y dicotómiCos), aplicado a una muestra ( $n=45$ ) constituida por sujetos residentes en un Centro de Inserción Social (CIS), esto es, un centro de régimen abierto, estado intermedio entre la ejecución de las penas privativas de libertad y la reincorporación a la sociedad, cuyo objetivo es potenciar las capacidades de inserción social de los sujetos. Los resultados revelan que frente a un centro penitenciario cerrado ordinario, el régimen abierto conlleva una perspectiva más positiva en los usuarios, reflejándose en factores como el apoyo social, el control emocional y en estados emocionales como los niveles percibidos de ansiedad, estrés y miedo.

Palabras clave: prisionalización; preso; adaptación; estado emocional

\section{ABSTRACT}

The inmate's emotional state facing the deprivation of liberty. Prisionalization requires an adaptation, both physical and psychological, that the inmate in a penitentiary institution must carry out for his adaptation to the prison context since, when he is deprived of liberty, there is an extremely abrupt change in his life. Thus, many and varied are the psychological effects suffered by subjects after a time residing in a penitentiary institution. In this sense, this study aims to analyze both the effects of imprisonment arouses, fundamentally in 


\section{ESTADO EMOCIONAL DEL INTERNO ANTE LA PRIVACIÓN DE LIBERTAD}

terms of the emotional states, in the individual and the impact that this experience generates on his development. For this, an ad hoc questionnaire of own elaboration has been used, made up of 46 items (multiple choice, Likert type and dichotomous), applied to a sample ( $\mathrm{n}=45$ ) made up of subjects residing in a Social Insertion Center, that is, a center with an open regime (an intermediate state between the execution of custodial sentences and reincorporation into society) whose objective is to enhance the subject capacities for social insertion. The results reveal that, compared to an ordinary closed penitentiary, the open regime implies a more positive perspective for users, reflecting on both factors such as social support, emotional control, and also on emotional states such as perceived levels of anxiety, stress and fear.

Keywords: prisionalization; inmate; adaptation; emotional state

\section{MARCO TEÓRICO}

La entrada en un Centro Penitenciario requiere una obligada adaptación. Según Echeverri (2010), el interno convive con una 'normalidad carcelaria', pero ésta no puede considerarse como una situación de bienestar o tranquilidad, ya que se naturaliza la adaptación a un ambiente tenso y exigente, lo que conlleva una importante inestabilidad emocional. Asimismo, de acuerdo con Ruiz (1999), el recluso está expuesto de manera cotidiana a diversas circunstancias que le obligan a realizar un sobreesfuerzo adaptativo. Esta adaptación es conocida como prisionalización.

Así, uno de los efectos más comprobados presentes en las personas durante su estancia en prisión es el estrés (con estados de ansiedad, depresión, etc.).

Según Andreu Mateu (2011), la determinación que ejerza la persona sobre su capacidad de adaptación ante la privación de libertad influirá en gran medida en la manera de afrontar la situación. De forma que si el interno es capaz de reunir los recursos necesarios para experimentar emociones positivas eficaces y hacer frente a la situación, el estrés quedará anulado. Sin embargo, si considera que es incapaz de superar la situación y las emociones que experimenta son negativas, creerá que no tiene la capacidad suficiente para lidiar con la situación y, por tanto, comenzarán las respuestas de estrés.

Por su parte, siguiendo a Barra (2004), el apoyo social constituye un principio de protección para paliar los efectos perjudiciales del estrés, de manera que la persona con apoyo social sólido aprecia los factores estresantes de manera menos peligrosa, puede afrontar las situaciones y problemas de forma más efectiva y se siente más segura. Además, el apoyo social se vincula con la red social de la persona, de forma que la escasez de apoyos hace que la persona sienta un mayor nivel de soledad y aislamiento.

De acuerdo con Arce, Fariña y Novo (2014), es preciso trabajar en pos de una intervención reeducativa que potencie el desarrollo emocional de los internos y, por ende, el autocontrol de sus emociones, ya que tienden a responder comportamentalmente con descargas emocionales, esto es, reducen la tensión a través de la expresión de sentimientos negativos.

\section{OBJETIVOS}

El objetivo general del trabajo es conocer el impacto emocional que provoca en la persona la privación de libertad.

Por su parte, los objetivos específicos persiguen:

Indagar el impacto emocional que provoca la prisionalización en internos en un Centro de Inserción Social.

Explorar la adaptación vivencial a un contexto carcelario en internos que se encuentran en un Centro Penitenciario de Medio Abierto.

Analizar el punto de vista de los internos ante el tratamiento dirigido a la gestión de las emociones.

\section{INSTRUMENTOS}

Se decide utilizar técnicas cuantitativas, siendo el cuestionario el instrumento elegido. Se elaboró un cuestionario ad hoc, conformado por 46 ítems, adaptados al nivel de alfabetización de los sujetos, ya que algunos no 
sabían leer ni escribir. Amén de tomar las medidas oportunas para la adaptación y adecuación de los mismos, se procura que los resultados no se vean afectados por juicios de valor u otros factores que interfieran.

Así, para disminuir la deseabilidad social que pudiera producirse se informó a los reclusos acerca del nulo impacto de sus respuestas sobre su situación penal y su estancia en la Institución. Además de hacer gran hincapié en que la información facilitada sería tratada de manera absolutamente anónima y confidencial y no transcendería al personal funcionario ni a la Dirección del Centro.

Los ítems iniciales del cuestionario están constituidos por preguntas de identificación, referidas a cuestiones sobre la situación personal y carcelaria de los sujetos.

Posteriormente, se utilizan ítems tipo Likert con el objetivo de saber cómo afecta el hecho de estar privados de libertad (tanto a reclusos que son primarios y, por tanto, es la primera vez que se encuentran en un contexto carcelario como a aquéllos que son reincidentes) en relación a los estados emocionales predominantes durante su estancia en prisión (nivel de apoyo social, ansiedad, estrés, control emocional).

A continuación, se plantean cuestiones dicotómicas para conocer cómo se han sentido con respecto a los diversos profesionales de la institución y las medidas que ésta ha implementado para que los internos conozcan sus derechos. También se tratan cuestiones relacionadas con el tratamiento recibido, tanto educativo como terapéutico.

Finalmente, se presenta un espacio abierto para que cada sujeto pueda expresar sentimientos, emociones 0 percepciones que han predominado en él al haber sufrido la experiencia de privación de libertad.

Para analizar la información se utilizó el paquete estadístico SPSS.

\section{MUESTRA}

La muestra seleccionada para llevar a cabo el estudio fue no probabilística, dadas las dificultades de acceso.

La muestra total analizada (ver Tabla 1) se compone de 45 sujetos, 41 hombres (91,1\%) y 4 mujeres (8,9\%), reclusos en un Centro de Inserción Social, por tanto, en régimen abierto. Su rango etario está entre 23 y 62 años, siendo la $=40,24$ y $S_{X}=10,34$. Respecto al 'estado civil', el 48,9\% (22 sujetos) tienen pareja -casados, unión de hecho y convivencia-y el 51,1\% (23 sujetos) están solos -solteros, separados o viudos-. En relación al 'país de nacimiento', el 95,6\% (43 sujetos) son de origen español y el 4,4\% de origen extranjero (2 sujetos, uno nacionalizado y otro con permiso de residencia). 


\section{ESTADO EMOCIONAL DEL INTERNO ANTE LA PRIVACIÓN DE LIBERTAD}

Tabla 1

Descripción de la muestra

\begin{tabular}{|c|c|c|}
\hline Sexo & Sujetos & Porcentaje \\
\hline Masculino & 41 & $91,1 \%$ \\
\hline Femenino & 4 & $8,9 \%$ \\
\hline Estado Civil & Sujetos & Porcentaje \\
\hline Casado/a & 7 & $15,6 \%$ \\
\hline Con pareja & 15 & $33,3 \%$ \\
\hline Soltero/a & 13 & $28,9 \%$ \\
\hline Separado/Divorciado/a & 10 & $22,2 \%$ \\
\hline \multicolumn{3}{|l|}{ País de Nacimiento } \\
\hline Español & 43 & $95,6 \%$ \\
\hline Extranjero & 2 & $4,4 \%$ \\
\hline \multicolumn{3}{|l|}{ Situación Legal } \\
\hline Nacionalizado & 1 & $50 \%$ \\
\hline $\begin{array}{l}\text { Con permiso de } \\
\text { residencia }\end{array}$ & 1 & $50 \%$ \\
\hline Media ( ) & $\begin{array}{l}\text { Desviación } \\
\text { Típica }\end{array}$ & Rango \\
\hline 40,24 & 10,34 & $23-62$ \\
\hline
\end{tabular}

Considerando la variable 'nivel de estudios' (ver Tabla 2), el 33,3\% posee Estudios Primarios y otro 33,3\% ES0/EGB. El 8,9\% cuenta con FP/Bachillerato, al igual que Estudios Universitarios, y, finalmente, el 15,6\% no tiene estudios.

Tabla 2

Nivel académico de la muestra

\begin{tabular}{ccc}
\hline Nivel de Estudios & Sujetos & Porcentaje \\
\hline Sin estudios & 7 & $15,6 \%$ \\
\hline Primaria & 15 & $33,3 \%$ \\
\hline ESO/EGB & 15 & $33,3 \%$ \\
\hline FP/Bachillerato & 4 & $8,9 \%$ \\
\hline Estudios Universitarios & 4 & $8,9 \%$ \\
\hline
\end{tabular}

La muestra está integrada mayoritariamente por internos de carácter primario, es decir el 60\% (27 sujetos) es la primera vez que son condenados y, por tanto, que ingresan en un Centro Penitenciario. Sin embargo, el $40 \%$ (18 sujetos) son reincidentes, es decir, han ingresado en diversas ocasiones (ver Tabla 3).

La Tabla 3 ilustra los delitos por los que han sido condenados: Ios internos condenados por delitos de 'robo y/0 hurto' constituyen el 34,9\% de la muestra, seguido de 'delitos de lesiones' que representan el 16,3\%, los delitos 'contra la salud pública' suponen el 11,6\%, la 'violencia de género' el 7\%, 'asesinato, homicidio imprudente, quebrantamiento de condena y agresión sexual', el 4,7\%, y, finalmente, delitos 'contra la seguridad vial', 'pertenencia a banda armada', 'estafa', 'malversación de fondos' y 'revelación de secreto' representan el 2,3\%.

Estos porcentajes corresponden al 95,6\% de la muestra (43 sujetos), ya que hay datos perdidos (2 sujetos), que no han contestado a dicha cuestión. 
Por lo que respecta a las sanciones (ver Tabla 3), el 75,6\% de la muestra no ha referido cumplir ningún tipo de sanción durante su estancia en prisión, frente a un 24,4\% que afirma haber cumplido algún tipo de sanción.

Respecto al tiempo pasado en una Institución Penitenciaria (ver Tabla 3), el 30\% de la muestra lleva 'menos de 6 meses' en prisión, el 17,5\% 'entre 3 y 4 años', el 15\% 'más de 6 años', el 10\% 'entre 2 y 3 años' y 'entre 4 y 6 años'. Los porcentajes más bajos corresponden a la estancia 'entre 1 y 2 años' (5\%) y 'entre 6 meses y 1 año' $(2,5 \%)$.

Estos resultados verifican una gran amplitud de condenas, así como internos tanto de carácter primario como reincidentes, además de procedentes de Centros Penitenciarios de carácter Ordinario en los que, debido a una progresión positiva, han conseguido ingresar en un Centro de medio abierto.

Tabla 3

Situación penitenciaria de los sujetos

\begin{tabular}{lcc}
\hline Clasificación & Sujetos & Porcentaje \\
\hline Primarios & 27 & $60 \%$ \\
\hline Reincidentes & 18 & $40 \%$ \\
\hline Delitos & 2 & \\
\hline Asesinato & $4,7 \%$ \\
\hline Homicidio Imprudente & 2 & $4,7 \%$ \\
\hline Delitos de lesiones & 7 & $16,3 \%$ \\
\hline Contra la seguridad vial & 1 & $2,3 \%$ \\
\hline Contra la Salud Pública & 5 & $11,6 \%$ \\
\hline Violencia de Género & 3 & $7 \%$ \\
\hline Robo y/o Hurto & 15 & $34,9 \%$ \\
\hline Quebramiento de condena & 2 & $4,7 \%$ \\
\hline Pertenencia a banda armada & 1 & $2,3 \%$ \\
\hline Estafa & 1 & $2,3 \%$ \\
\hline Agresión Sexual & 2 & $4,7 \%$ \\
\hline Malversación de Fondos & 1 & $2,3 \%$ \\
\hline Revelación de secretos & 1 & $2,3 \%$ \\
\hline Valores perdidos & 2 & \\
\hline Cumplimiento de sanciones & & \\
\hline Sí & 11 & $24,4 \%$ \\
\hline No & 34 & $75,6 \%$ \\
\hline Tiempo de condena & \multicolumn{2}{|c}{} \\
\hline Menos de 6 meses & 12 & $30 \%$ \\
\hline Entre 6 meses y 1 año & 1 & $2,5 \%$ \\
\hline Entre 1 y 2 años & 2 & $5 \%$ \\
\hline Entre 2 y 3 años & 4 & $10 \%$ \\
\hline Entre 3 y 4 años & 7 & $17,5 \%$ \\
\hline Entre 5 y 6 años & 4 & $10 \%$ \\
\hline Entre 5 y 6 años & 4 & $10 \%$ \\
\hline Más de 6 años & 6 & $15 \%$ \\
\hline Valores perdidos & 5 & \\
\hline
\end{tabular}

La variable 'formación académica y laboral' de los internos durante su permanencia en prisión se presenta en la Tabla 4. Apreciamos cómo más de la mitad de la muestra (el 57,8\%) ha realizado algún tipo de trabajo 


\section{ESTADO EMOCIONAL DEL INTERNO ANTE LA PRIVACIÓN DE LIBERTAD}

durante su estancia. Esto podría deberse a la preferencia de ocupación de su tiempo libre, ya que muchos afirman sobrellevar mejor la estancia si ocupan su tiempo.

Por su parte, la formación académica en prisión (ver Tabla 4) alcanza, igualmente, porcentajes elevados. Así, el $55,6 \%$ de los sujetos realiza o ha realizado algún tipo de formación que puede aumentar las posibilidades de integración/reinserción en el exterior.

Tabla 4

Presentación de la ocupación durante el periodo de estancia en prisión

\begin{tabular}{ccc}
\hline Trabajo & Sujetos & Porcentaje \\
\hline Sí & 26 & $59,1 \%$ \\
\hline No & 18 & $40,9 \%$ \\
\hline Valores Perdidos & 1 & \\
\hline Estudios & & $52,3 \%$ \\
\hline Sí & 23 & $47,7 \%$ \\
\hline No & 21 & \\
\hline Valores Perdidos & 1 &
\end{tabular}

\section{RESULTADOS Y DISCUSIÓN}

Para el mejor entendimiento de la representación y análisis de los datos obtenidos en el cuestionario, en primer lugar, se agrupan por variables, es decir se analizan los datos en relación con las variables objeto de investigación (apoyo social, control emocional, estado depresivo, ansiedad y estrés). En segundo lugar, las respuestas se agrupan en tres categorías siendo éstas: 'en desacuerdo' con las cuestiones tratadas, 'ni en desacuerdo ni de acuerdo' y, por último, 'de acuerdo' con las mismas.

La primera variable analizada se refiere al 'apoyo social' que experimenta el interno al encontrarse privado de libertad. Dentro de esta variable se especifican diversas cuestiones.

Así, en primer lugar (ver Figura 1), se representan cuestiones relacionadas con la ayuda que pueden obtener de las personas más allegadas en relación al tratamiento de sus problemas, es decir el apoyo que pueden tener de ellas para tratar de apaciguar el sentimiento de malestar.

Los resultados muestran que mayoritariamente creen poseer dicho apoyo, puesto que el 59,05\% de la muestra posiciona su valor de acuerdo con la afirmación expuesta, es decir se sitúa en un enfoque positivo con respecto al sustento de las personas más cercanas. Este alto porcentaje podría deberse a la posibilidad de estar efectivamente presentes en su contexto social ya que, al encontrarse en régimen abierto, realizan salidas al domicilio de acogida que la familia 0 algún miembro de su red social pueda facilitar para acoger al sujeto en sus salidas al exterior. El 28,40\% alegan no estar ni de acuerdo ni en desacuerdo y el 12,45\% se posiciona en desacuerdo con la cuestión, por lo que podríamos concluir que dicho porcentaje de personas sienten no tener apoyo de este tipo. En estos casos, la lejanía geográfica del Centro respecto a su lugar de origen o la situación económica familiar pueden dificultar el mantenimiento del contacto con la red social. 
Figura 1

Representación del apoyo social recibido por las personas más allegadas al individuo

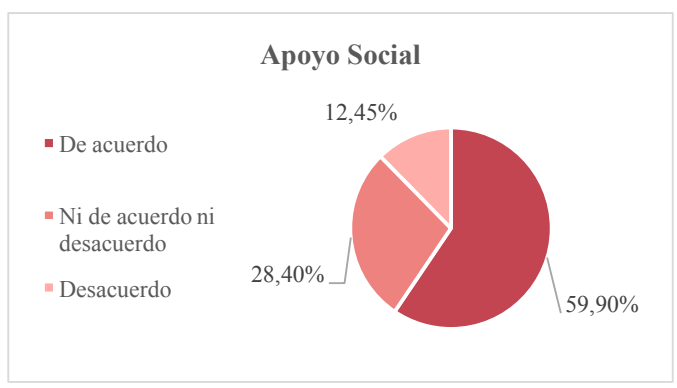

En segundo lugar, se incorporan los resultados obtenidos respecto a la existencia de personas que proporcionan dicho apoyo, indagando sobre sus relaciones personales. Se pretende determinar si el sujeto percibe que realmente existe una preocupación hacia él por parte de su familia y amigos (ver Figura 2).

De acuerdo con los resultados, se puede percibir el alto nivel de percepción hacia la tenencia de apoyo social por parte de los suyos, puesto que el $82,30 \%$ afirma estar de acuerdo con las cuestiones expuestas que evalúan la preocupación hacia los sujetos durante su estancia en prisión por parte de sus seres queridos. Solo el 9,45\% refiere no estar ni en desacuerdo ni de acuerdo y el 8,25\% afirma estar en desacuerdo, por lo que no creen tener personas cercanas que muestren preocupación por ellos.

Figura 2

Exposición del apoyo social percibido concretando en el sustento que aporta la familia y amigos

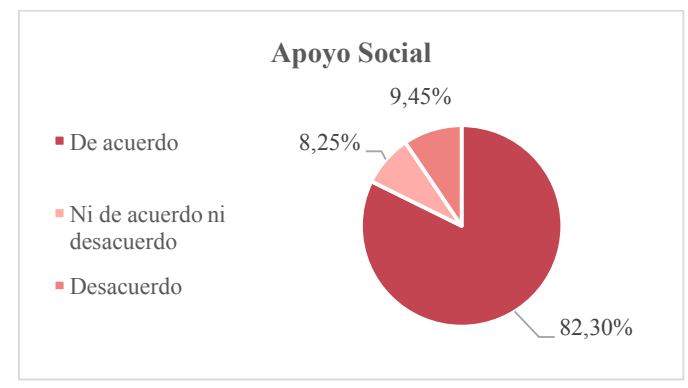

En segundo lugar, se analiza otra de las variables: el control emocional, específicamente 'el control de la frustración y el enfado'al ser esencial durante su estancia en prisión para conservar el bienestar personal y para la autogestión de acciones conductuales. Las afirmaciones para analizar estos factores se fundamentan en el control que las personas creen ejercer ante problemas y/o situaciones que generan ira.

Como observamos en la Figura 3, el 57,42\% está en desacuerdo con las afirmaciones descritas, es decir refieren tener la capacidad para controlar el enfado y la frustración. Sin embargo, el 27\% afirma estar de acuerdo con las afirmaciones expuestas, circunstancia que, a pesar de ser un porcentaje más reducido, refleja la carencia o dificultad de control de sus estados emocionales. Siendo el 19,52\% restante aquellos sujetos que no están ni en desacuerdo ni de acuerdo. 


\section{ESTADO EMOCIONAL DEL INTERNO ANTE LA PRIVACIÓN DE LIBERTAD}

Figura 3

Descripción del control emocional percibido ante el enfado y la frustración

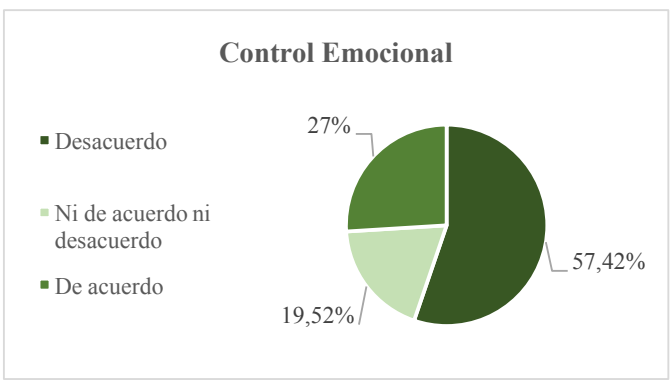

Teniendo en cuenta investigaciones previas, como la de Arce, Fariña y Novo (2014), el recluso tiende a renunciar a la solución efectiva de problemas y presenta una tendencia a solventarlos expresando emociones negativas. Sin embargo, la mayoría de la muestra de nuestro estudio refiere controlar los distintos estados de ánimo. Este hecho podría deberse a la deseabilidad social; es decir, al encontrarse en la última etapa penitenciaria podrían actuar de una manera controlada, respondiendo lo que se espera de ellos. Así podría verse reflejado el recelo a mostrar una conducta no deseada y, en consecuencia, a marcar aspectos negativos que perjudiquen su estancia en prisión, así como su reinserción en la sociedad, ya que la amenaza de un retroceso a un Centro de Régimen Ordinario está siempre presente.

Dentro de esta variable -control emocional- se pretende indagar también en el control que tienen los sujetos del miedo experimentado al ingresar en un entorno tan desconocido y estereotipado como la cárcel. Este control del miedo puede realizase exhibiendo, justamente, lo contrario: una conducta agresiva para enfrentarse al contexto tan hostil.

En este sentido, el 56\% de la muestra se encuentra en desacuerdo con las cuestiones relativas al miedo, alegando no sentir emociones de este tipo ante la entrada y estancia en prisión. Frente al 24,9\% que muestra no estar ni de acuerdo ni desacuerdo y el 19,10\% que afirma estar de acuerdo, éstos manifiestan haber sentido miedo/pánico (ver Figura 4).

Figura 4

Representación del control emocional que ejerce el sujeto ante el miedo

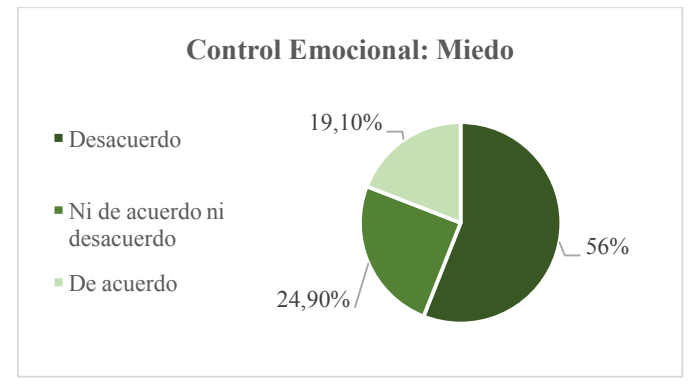

Estos resultados podrían deberse a diversos factores. Como hemos referido anteriormente, uno de ellos podría ser el encubrimiento de este tipo de emociones con el objetivo de disminuir la vulnerabilidad ante otros sujetos e, incluso, ante la Institución. También por la permanencia en el contexto actual, ya que el CIS constituye 
un puente intermedio entre la cárcel y la libertad, donde las conductas de carácter agresivo son infrecuentes. Sin embargo, el porcentaje que muestra experimentar dichas emociones podría deberse a un escaso tiempo de permanencia en el mismo, con el consiguiente desconocimiento del centro, la inseguridad u otros factores similares.

Finalmente, la Figura 5 refleja el malestar asociado a la preocupación por situaciones familiares externas, sobre las que pierden el control una vez ingresan en prisión. Los resultados revelan preocupación al respecto. Ello puede repercutir en la falta de control ante sus pensamientos y sentimientos y menguar su bienestar. Así, el $64,4 \%$ de los sujetos afirman estar de acuerdo con la preocupación por situaciones y circunstancias fuera de la Institución, el 20\% muestra su desacuerdo y el restante 15,6\% alega no estar ni de acuerdo ni desacuerdo.

Figura 5

Control emocional que poseen los sujetos ante la preocupación por situaciones y circunstancias externas a la prisión

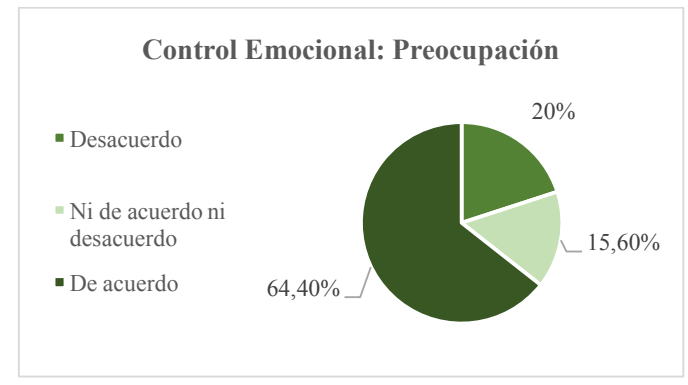

A continuación, se comentarán los resultados obtenidos en referencia a las variables relacionadas con 'pensamientos/conductas depresivos' propuestas en el estudio. Puede clasificarse en dos bloques diferenciados: el estudio de la percepción que posee el individuo sobre cómo ha sido su vida pasada y cómo es su vida actual, si cree que tiene sentido y se encuentra motivado e ilusionado y, por otra parte, se muestran los resultados obtenidos en relación a la iniciativa que creen tener para realizar actividades 0 acciones que deben llevar a cabo en su vida.

En referencia a los ítems dirigidos a sondear la percepción que tienen los sujetos de su vida pasada (ver Figura 6), los resultados muestran una actitud mayoritaria negativa, afirmando que ha sido injusta y dura. Así, el $64,5 \%$ de la muestra se muestra de acuerdo. El 17,7\% refiere no estar ni de acuerdo ni desacuerdo y el restante $17,8 \%$ manifiesta estar en desacuerdo y, por tanto, tiene una visión positiva sobre su vida pasada.

Figura 6

Percepción sobre su vida pasada

Pensamientos Depresivos
- Desacuerdo
$\begin{aligned} & \text { Ni de acuerdo ni } \\ & \text { desacuerdo } \\ & \text { - De acuerdo }\end{aligned}$




\section{ESTADO EMOCIONAL DEL INTERNO ANTE LA PRIVACIÓN DE LIBERTAD}

A continuación, los resultados obtenidos sobre su vida presente, en comparación con los obtenidos sobre su vida pasada, es decir si experimentan tristeza o si no le encuentran sentido a su vida. Los resultados (ver Figura 7) presentan un porcentaje de respuestas bastante igualado, ya que el 49,6\% alegan estar en desacuerdo con dichas afirmaciones (por lo que no experimentan tristeza o sentimientos que puedan relacionarse con estados depresivos y refieren sentir una gran motivación vital). Sin embargo, el 36\% se confiesa de acuerdo con estas afirmaciones (refiere experimentar estados depresivos y no encontrar estímulos en su vida). El porcentaje restante refleja aquéllos que se posicionan de manera neutral: el 14,3\% de los sujetos no están ni de acuerdo ni en desacuerdo con estas afirmaciones.

Figura 7

Percepción sobre su vida actual

Pensamientos Depresivos
= Desacuerdo
$\begin{aligned} & \text { Ni de acuerdo ni } \\ & \text { desacuerdo }\end{aligned}$
= De acuerdo

De igual manera, cuando se analiza la iniciativa y la motivación que poseen con respecto a la realización de actividades (ver Figura 8), se aprecian resultados similares a los referidos anteriormente, ya que el 48,8\% muestra no posee dificultad para realizar cualquier actividad y muestra motivación por su vida diaria, mientras el $34,8 \%$ percibe cómo un estado de ánimo caracterizado por pensamientos negativos dificulta las actuaciones en su día a día. El resto de los participantes (16,3\%) no está ni de acuerdo ni en desacuerdo con estas cuestiones.

Figura 8

Representación de la motivación que tienen en su vida

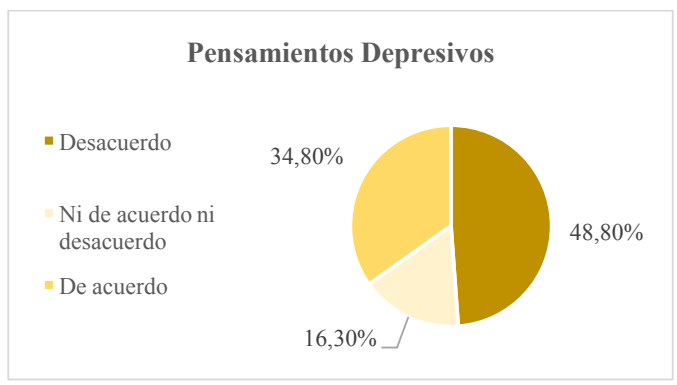

Por último, se procede a analizar las cuestiones relacionadas con 'el estrés y la ansiedad'. Las afirmaciones que reflejan algunas vivencias que la persona puede haber experimentado relacionadas con estos estados emocionales son: tener la boca seca, sentir presión en el pecho, poseer dificultad para respirar, etc.

Los resultados obtenidos se reflejan en la Figura 9, predominando aquellas personas que muestran su desacuerdo ante estas cuestiones: el 45,5\% de la muestra alega no sufrir este tipo de manifestaciones. Sin embargo, 
se puede comprobar cómo el resto de los porcentajes no son excesivamente bajos con respecto al anterior, por lo que, a pesar de ser un porcentaje menor, el 30,2\% muestra su acuerdo y, por tanto, afirman haber experimentado estrés/ansiedad, siendo el restante 24,30\% aquellos que refieren no estar ni de acuerdo ni en desacuerdo.

Figura 9

Representación de las manifestaciones de estrés y ansiedad que han experimentado

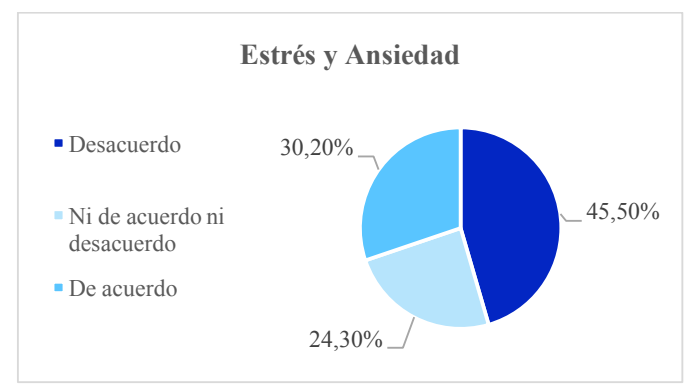

El estrés y la ansiedad pueden estar presentes por diversas razones vinculadas tanto a la privación de libertad, como a la expectativa de una ya cercana libertad, lo cual también puede generar estrés por la incertidumbre respecto a su reinserción.

A continuación, se exponen los resultados relativos a la opinión de los sujetos sobre el tratamiento recibido por parte de la Institución.

En primer lugar, respecto a las cuestiones relacionadas con la información recibida por parte del equipo institucional acerca de los derechos y deberes del interno, el 59,1\% de la muestra refiere haber sido informado de sus derechos y deberes, frente al 40,9\% que indica no haber sido informado.

En esta misma línea está lo afirmado en relación con los ítems referidos a la información aportada por parte de la Institución sobre la atención que recibirían o podrían obtener de los profesionales: el 54,4\% afirma haber recibido dicha información y, por el contrario, el 45,5\% restante asegura no haber recibido ningún tipo de información al respecto.

Por tanto, a pesar de ser mayoritario el porcentaje que afirma haber recibido información, un porcentaje considerable afirma no haberla recibido. Esto podría deberse a ciertas carencias por parte del Sistema Penitenciario pero también debe contemplarse con prudencia porque la Institución en sí misma constituye un elemento manifiestamente no grato para los participantes, pudiendo no recordar el hecho, distorsionarlo o mentir sobre el mismo.

Con respecto a las actividades desarrolladas durante su estancia en prisión, el 68,2\% de los sujetos manifiestan haber realizado alguna actividad de carácter terapéutico o educativo, mientras que el 31,8\% refiere no haber realizado ninguna actividad tal. Respecto a la realización de actividades encaminadas a disminuir el estrés, ansiedad o nerviosismo que la privación de libertad puede acarrear, el 70,5\% de los sujetos refiere no haber participado en ninguna actividad relacionada con el control emocional, frente al 29,5\% que asegura haber realizado actividades para ayudar a disminuir dichos estados emocionales.

A continuación, se tratan cuestiones relacionadas con la ayuda ofrecida por parte del personal funcionario para el control de emociones y la búsqueda del bienestar personal. Al respecto, se aprecia una visión negativa hacia los profesionales: el $84,1 \%$ de los sujetos afirman que los profesionales no le proporcionan ayuda para controlar dichas emociones, frente al 15,9\% que manifiestan haber recibido apoyo en tal sentido por parte del personal funcionario. Ello podría deberse a la escasez de recursos y programas dirigidos a paliar los efectos, sobre todo en el ámbito emocional, que provoca la privación de libertad. 


\section{ESTADO EMOCIONAL DEL INTERNO ANTE LA PRIVACIÓN DE LIBERTAD}

Finalmente, analizamos los posibles estereotipos sobre la cárcel y, a su vez, si la visión de los sujetos cambia una vez ingresan en prisión y, por ende, si se modifica también la visión sobre el propio colectivo recluso.

En este sentido, el 79,5\% de los sujetos consideran que los medios de comunicación (televisión, radio, etc.) deforman la realidad que se vive en prisión, mostrándola más hostil y amenazadora de lo que es y el 72,7\% afirma que su visión cambió una vez entraron en el Centro e, incluso, aclaran que, a pesar de ser una experiencia dura, no se corresponde con la imagen estereotipada que la sociedad tiene.

\section{CONCLUSIONES}

Se ha podido observar que en la muestra aparecen altos porcentajes de bienestar personal percibido, probablemente asociados a estar en un Centro de régimen abierto. Los factores estudiados, tales como el apoyo social, el control emocional y saber lidiar eficazmente con diversos estados como la ansiedad, el estrés, el miedo, etc. reflejan una perspectiva mayormente positiva de sí mismo y, a pesar de la deseabilidad social y del recelo por las repercusiones potenciales de una conducta indeseada, los resultados reflejan un contexto más próximo a la visión educativa que el adoptado en los centros cerrados.

Además, se aprecia una significativa carencia percibida por parte de los internos en cuanto al asesoramiento, información y educación sobre el manejo de los estados emocionales negativos que pueden sufrir a lo largo de la experiencia de privación de libertad.

Así, la educación se revela esencial para llevar a cabo acciones reeducativas que ayuden a la reinserción social del individuo, trabajando la motivación para el cambio y la prevención de la reincidencia, siendo imprescindible para ello el trabajo en el ámbito emocional, el autocontrol, la eficiencia, la responsabilidad, etc.

\section{REFERENCIAS BIBLIOGRÁFICAS}

Andreu Mateu, M.S. (2011). Un protocolo de tratamiento psicológico para los trastornos adaptativos apoyado por un sistema de realidad virtual: análisis de su eficacia a través de un estudio controlado. Tesis Doctoral. Castellón: Universidad Jaume I.

Arce, R., Fariña, F. y Novo, M. (2014). Competencia cognitiva en penados primarios y reincidentes: Implicaciones para la reeducación. Anales de Psicología, 30 (1), 259-266.

Barra, E. (2004). Apoyo social, estrés y salud. Psicología y Salud, 14 (2), 237-243.

Echeverri, J.A. (2010). La prisionalización, sus efectos psicológicos y su evaluación. Pensando Psicología, 6 , 157-166.

Ruíz, J.R. (1999). Estrés en prisión y factores psicosociales. Revista Colombiana de Psicología, 8 (1), 120-130. 